\title{
THE INTELLIGENT SYSTEMS FOR LANGUAGE PHONETIC INTERFACES
}

\author{
Natalia Yanova \\ Altai State University, Lenina 61, Barnaul, Russia
}

\begin{abstract}
The project aims to develop intelligent natural language analysis tools. The aim of the project is to formalize the interpretative approach in the study of perception and understanding of language interfaces. The project implements an expert system of psychophonetic analysis in the field of onomastics. The system is designed for psychological diagnostics of anthroponyms in the Russian language. The project offers a research technique for the psychophonetic study of naming in natural language. The project discusses the formalization of psychophonetic functions of the mental lexicon. The subject of the analysis is the psychological structure of phonosemantic decoding of nouns. A psychometric model is proposed for calculating the interpretation functions of the psychophonic meaning of names. The research solution includes mobile software application for IOS and Android (smart-test 'What's Name').
\end{abstract}

\section{KEYWORDS}

Natural Language Processing, Sentiment Analysis, Expert systems, Psychophonetics

\section{INTRODUCTION}

The development of natural language intellectual analysis systems sets the prospect of a convergence of the humanities, natural sciences and engineering. Calculation and modeling of Hidden Structure and Function in the Lexicon becomes a common subject of analysis of neurocomputer, neurocognitive and neurolinguistic approaches to language processing in living and artificial systems (Sharp at al. 2017). This is facilitated by methods of cognitive-mathematical modeling in the study of perception and understanding of a language on a computational basis. The goal is to formalize the principles and functions of interpretation in a language (Strauss at al. 2018). The measurement of interpretation functions (language comprehension / generation / ambiguity / predictability, attribution) is a prospect for semantic information processing methods. At the same time, the convergence of terms and theories in attempts to formalize the principle of interpretation is slower than methods and techniques (Suprun, Yanova \& Nosov, 2015).

Cognitive and mental processes of language understanding find various technical implementations in large related areas: natural language processing (NLP), natural language understanding (NLU), knowledge representation, natural language generation (NLG). The problem of interpretation as the complexity of semantic decoding affects narrower areas of analysis: Discourse Integration (semantics of context), Pragmatic Analysis (pragmatics of experience and situation), Emotion Detection (recognition of emotions), Sentiment Analysis (analysis of text tonality), Opinion Mining (mental response), Text Mining (intellectual processing of text). The 'super-task' of such studies is to measure not so much the accuracy and errors of interpretation as the assessment of the human factor and psychology (Chen at al. 2018).

The evaluation functions of understanding and interpretation in the human language pose a challenge for finding new engineering solutions in Human Interaction and Emerging Technologies. This is evidenced by the rapid development of psychologically oriented technical tracks: Digital Human Modeling, Human-Centered Design, Emergent Properties of Human Behavior, Usability and User Experience, Ubiquitous \& Affective Computing, Needs and Emotions for Intelligent Systems. Among the tools of language analysis with 'psychological calculations': test expert systems, semantic modeling systems, recognition and decoding systems, dialog systems. Modern smart-applications combine and expand their capabilities. 
The engineering development of such systems takes into account human psychology (Andina et al. 2015). Increasingly, the question is being raised about modeling the emotional processes of cognition (intentional and evaluative). From word-based computing, we are moving to computing of perceptions, goals, intentions, emotions, attitudes. This is the area of 'language personality' psychodiagnostics. Psychodiagnostics and psychometrics in the cognitive digital revolution are becoming part of the information, cognitive and neurotechnologies. Intelligent systems in everyday life become mental assistants. Today they provide access not only to information (Data analysis) and knowledge (Data mining), but also to cognition (Opinion mining). Such expert systems developed on the basis of the Theory of Mind (Wellman \& Liu, 2004) in psycholinguistics proves the differences between verbal and mental lexicon as they measure not only the external meanings of a word, but also internal ones (mental representation).

\section{METHODOLOGY AND METHOD}

This project presents an expert system for the psychological analysis of onomastics of the Russian language. The goal is to analyze the perception of sound nomination in natural language. The system implements the 'Opinion mining' data format with an extended class of tasks, 'Emotion Detection' and 'Sentiment analysis'.

The field of such research belongs to psychophonetics that connects the psychology of perception with the phonology and phonetics of language. The perceptual side of speech sounding is the essence of psychophonetic analysis. In this method, an attempt is made to combine phonemic and semantic features of the language implementation in speech. We study 'psychophonetic of language and 'phonosemantic of speech'. The method includes the diagnosis of the euphony of the phonemes and allophones sounding. If a phoneme is a language abstraction, then an allophone is a specific implementation of a phoneme. A native speaker can always determine a mentally significant set of allophones in the implementation of phonemes. We investigate the psychology of phoneme perception in allophone types (basic, combinatorial, positional) to predict the evaluation of the lexicon sounding in a particular mentality. The totality of all possible phoneme allophones in a language is called distribution, and their evaluation and interpretation is called attribution.

The search for stable sound combinations of phonemes is of great interest to linguists, starting with Charles Osgood and his method of semantic differential. His idea of psychophonetic meaning indicates a motivated connection between sound and semantics and explains the effects of sound image and sound symbolism of language in speech. The psychophonetics of the name has linguistic boundaries, since languages have different phonemic inventory and different phoneme distribution and attribution of allophones. For this reason, the psychology of naming makes sense only for the native language and is relevant with borrowed words. Our project explores the mental perception of proper names (both native and foreign) in Russian. The object of psychophonetic analysis in onomastics is the phonosemantic code of the name. The research question concerns the decoding function of psychophonic meaning. The structure of denotate, designate and connotate is considered. The objective of the study is to evaluate the psychology of sounding a name in a mental lexicon. The research method is psychodiagnostics of anthroponyms (proper names). The engineering solution is a mobile application (smart test). Let's consider the design of the test to evaluate the perception of the person's name in the Russian language.

\section{TECHNOLOGY AND APPLICATION}

Our application is based on computing with names and intended for the psychological analysis of the consonance of the name/patronymic/surname. The application combines both a test function and a decoder function for naming. The mobile application includes versions for IOS and Android (Tools SNY Research Group). Instruction manual for user is: 'Have you ever tried to come up with a name that chimes with your chosen audience?'Smart-test allows you to get the access code to the enigma of painting a picture with a name in your mind. Mobile application test any name to ascertain the perception of its sound in Russian choosing the right combination of first name-surname, first name-patronymic or surname-first name- patronymic, and thus create new forms of names and forms of address. This is usability of applied linguistics in everyday life. Takes a step from discovery into the science of puzzles - what to call a child, 
how to choose an iconic slogan, what form of address is preferable. You come up with the questions - the program comes up with the answers. Simply say or write down a name and obtain its unique underlying code and thus an insight into its frequency interpretation.

The result of test is a phonosemantic code for the name and a psychophonic profile for a specific variant of naming. The test provides a quantitative and qualitative assessment of the interpretation of the name, taking into account socio-cultural and mental stereotypes. Assessing the likely perception of the sound of the name includes the computational indices of the emotional and mental code. Integral assessment of naming is a condition of "acceptance-rejection" of a name for native speakers.

The calculation model is obtained from research on the assessment of psychophonetic equipment by native speakers of the Russian language. Phonemic inventory includes 46 phonemes, 33 letters, 56 stable "stressed/unstressed" combinations, 260 stable "soft/hard" combinations on 30 bipolar scales. An experimental study included testing native Russian speakers (1000 people) for the perception of allophones (basic, combinatorial, positional) on 30 scales. As a prototype, we used the Zhuravlev approach (Zhuravlev, 1981). Taking into account the frequency of sound in a language and a word, the place of sound and its stress, its method offers a formula for calculating the phonetic significance of a word:

$$
F=\frac{\sum_{i} f_{i} k_{i} l_{i} \frac{P_{\max i}}{P_{i}}}{\sum_{i} k_{i} l_{i}}
$$

$P_{\max i}$ - maximum frequency of the sound in a given word, $P_{i}$ - frequency of the sound in a word, $f_{i}$ - the value of the semantic differential of the phoneme on the each $l_{i}$ scale, $k_{i}$ - the value of the place of sound (the first/ not the first phoneme in the word) and its stress (the stressed/ unstressed phoneme in the word).

We use this formula for Russian letters, except for the calculation $f_{i}$. In our case, instead of Osgood's semantic differential method on a linear scale, the method of semantic analysis on a relativistic scale is used. The relativistic scaling method takes into account not only the intensity, but also the rigidity of the assessment in mentality. The value of function $f_{i}$ has not linear, but angular coordinates. The measurement method for $f_{i}$ in the relativistic scale is described in the author's publication (Yanova, 2016).

The computational algorithm of phonetic code indexes:

- Calculation $f_{i}$ for $i$ - scale, $N_{i}$

- Selection scales with $f_{i}>0,5, N_{j}$

- Volume Index $V=N_{j} / N_{i} * 100 \%$

- Determination of the reference and experimental valence of the sign for the $i$ - scale, $K_{i}$ (number of theoretical matches/key to the test) and $K_{j}$ (number of experimental matches/ key to the test).

- Consonance Index $C=K_{j} / K_{i} * 100 \%$

- Sound Index $S=\sum_{j=1}^{K j} f_{j} / \sum_{i=1}^{K i} f_{i} * 100 \%$

The calculation of mental code indexes is based on regression models (stepwise regression) from a list of 30 scales as independent variables for each dependent variable (mental code index).

\section{RESULTS}

Computing parameters of smart-test describe the phonetic and mental interpretation for a variant of nomination. Phonetic interpretation includes indexes of the phonetic code and reliable scales of the psychophonetic profile. Mental interpretation includes indexes of mental stereotypes of sound perception. Consider an experimental analysis example for a native and foreign name (Table 1, Figure 1). 
Table 1. Consonance of first and last foreign name in Russian

\begin{tabular}{lr|lr}
\hline 'Donald Trump' & $f_{i} k$ & 'Emmanuel Macron' & $f_{i} k$ \\
\hline heavenly- earthly & 9,65 & calm-anxious & $-6,83$ \\
\hline feminine-masculine & 9,16 & cheap-expensive & 5,21 \\
\hline light -heavy & 7,86 & cowardly-brave & 5,15 \\
\hline cowardly-brave & 7,60 & add-on (free $)$ & 5,08 \\
\hline frivolous-serious & 7,50 & clean-dirty & $-4,66$ \\
\hline child-adult & 6,43 & feminine-masculine & 4,29 \\
\hline stable-volatile & $-6,98$ & stable-volatile & $-4,30$ \\
\hline obedient-moody & $-5,28$ & heavenly-earthly & 4,14 \\
\hline calm-anxious & $-5,05$ & aristocratic-democratic & $-3,84$ \\
\hline sick-healthy & 4,58 & obedient-moody & $-3,79$ \\
\hline light-dark & 4,48 & safe-scary & $-3,60$ \\
\hline gentle-coarse & 4,31 & frivolous-serious & 3,49 \\
\hline strict-free & $-4,88$ & ugly-beautiful & 3,37 \\
\hline cheap-expensive & 3,78 & sick-healthy & 2,96 \\
\hline Volume Index & 36,70 & Volume Index & 23,30 \\
\hline Consonance Index & 70,00 & Consonance Index & 63,30 \\
\hline Sound Index & 65,20 & Sound Index & 80,40 \\
\hline Gender Code & male & Gender Code & male \\
\hline Sex Code & sexual & Sex Code & sexual \\
\hline Adult Code & adult & Adult Code & adult \\
\hline Native Code & uncertain & Native Code & uncertain \\
\hline Connotation Code & neutral & Connotation Code & neutral \\
\hline
\end{tabular}

Computing parameters for phonetic code (\%):

Volume Index (volume of the name) - index of the name recognition in Russian. This index characterizes the speed and ease of name recognition in the mentality. Volume index defines the name recognition code in the language of native speakers. It calculates the denotative value of the name (states of attributes by the denotation function).

Consonance Index (consonance of the name) - index of the name euphony in Russian. This index shows how expressive the name sounds to native speakers. It calculates the connotative value of the name. The index is determined by the number of matches of name positive attributions with the phonetic norm.

Sound Index (power of the name sounding) - index of the cultural influence. This index expresses the strength and stability of the name interpretation in Russian. It reveals the correspondence between the sound form and the lexical content of the name meaning. The index calculates the designative value of the name. Computing parameters for mental code $(\%)$ :

Gender Code - compliance with gender stereotype of the name perception (masculine/female/ androgynous - no gender stereotype of sounding). Sex Code - sexual appeal in the sound of the name.

Adult Code - age stereotype of the name sounding: perceived as an adult, carries an assessment of respectful treatment, suggests distance in communication; perceived as childish, has an assessment of ease and informality in communication, assumes personal, not official distance in communication.

The specified code parameters are calculated on the basis of correlations with the corresponding scales (male, female, adult, childish, sexual). Name calculation integral parameters:

Native Code - matching the sound of the name to the mentality of the native language: native/foreign (identification/ no identification with the Russian mentality). This parameter defines the Russian language phonosemantic in the sound of the name. It is calculated as a correlation with the "own or other" scale. Connotation Code - valiancy of the name sounding in Russian (positive, negative, neutral). The parameter is calculated by correlating the integral characteristics with the positive pole of the scales in the phonetic norm.

An experimental example of a computational model for native name in Russian: 

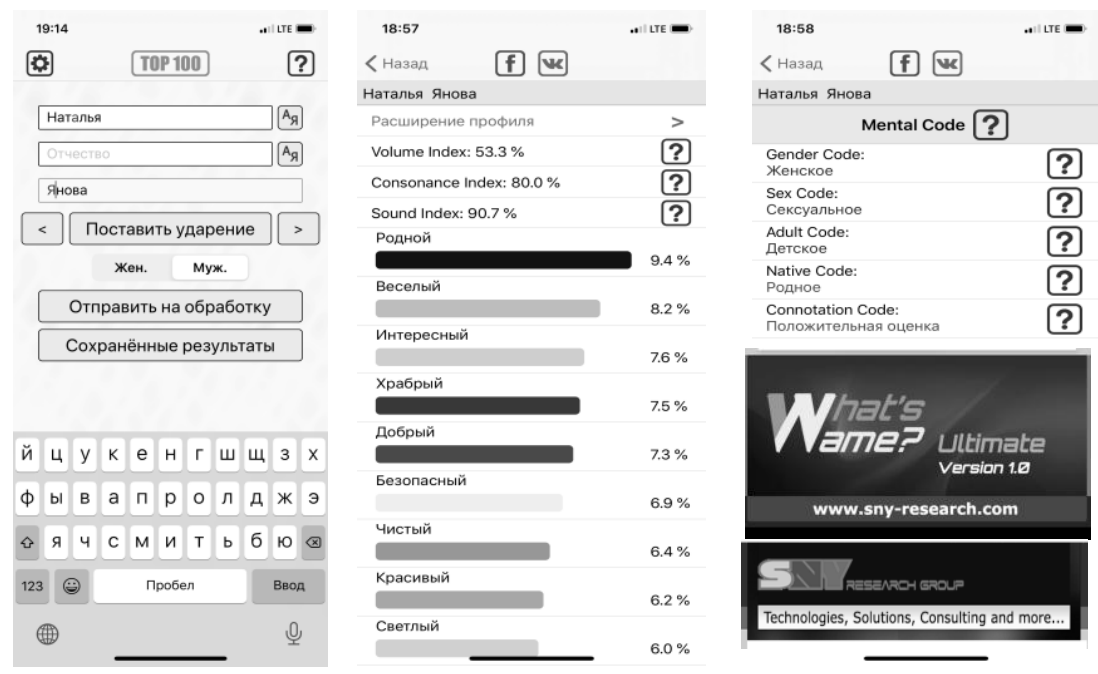

Figure 1. Mobile application: experimental example

\section{CONCLUSION}

The method for computing the perception and interpretation of names in Russian language allows explaining the hidden structure between lexicology and phonetics, to discover the semantic nature of language gaps associated with psychological function of voice information encoding. The computational model is carried out according to the sign structure that allows calculating such functions of name interpretation as recognition, euphony and influence. Checking the correspondence of the name to the basic mental stereotypes allows finding out the attitude to the name of a native Russian speaker. The significance of the work is connected with the theoretical and practical issues of onomastics, and is of particular significance for intercultural communication. The study helps to reveal for psychological differences between the nomination in different languages and cultures. It proposes not only the psychophonetic profile but also the structure of the psychophonetic meaning of the name. The user can independently assess the degree of congruence of perception and understanding of any name in Russian language. The know-how of the expert system consists of analyzing not only phonemes but also allophones. The system allows us to analyze of various options for nominations and choose among them the most successful one. Serious scientific theories and computational methods are becoming in demand for solving everyday problems.

\section{REFERENCES}

Andina, G. et al, 2015. Phonology and arithmetic in the language-calculation network. In Brain and Language, Vol. 143. pp. 97-105.

Chen, A. at al, 2018. Are lexical tones musical? Native language's influence on neural response to pitch in different domains. In Brain and Language. Vol. 180-182, pp. 31-41.

Sharp, B., Sedes, F., Lubaszewski,W., 2017. Cognitive Approach to Natural Language Processing. ISTE Press, Elsevier, London/Oxford, United Kingdom.

Strauss G.P., Allen D. N., 2008. Emotional intensity and categorization ratings for emotional and nonemotional words. In Cognition and Emotion, Vol.22, No.1, pp.114-133.

Suprun, A., Yanova, N., Nosov, K. 2015. Semantic Analysis of Meanings in Psycholinguistics. In Journal of Russian and East European Psychology, Vol.51, No.5, pp.170-172.

Tools SNY. Available at: http://www.sny-research.com/ (Accessed: 20 June 2020).

Wellman H.M., Liu D., 2004. Scaling of theory-of-mind tasks. In Child Development. Vol. 75, No.2, pp.523-541.

Yanova, N., 2016. Relativistic psychometrics in subjective scaling. In Elsevier Procedia Computer Science, V.102, pp. 82-89.

Zhuravlev A.P., 1981. Phonetic meaning. Publishing house of Leningrad State University, Leningrad, Russia. 